\title{
A Study of MCA Learning Algorithm for Incident Signals Estimation
}

\author{
Rashid Ahmed \\ National Technical University of Athens \\ School of Electrical and Computer Engineering \\ 9, Iroon Polytechniou St., 15773 Athens, Greece
}

\author{
John N. Avaritsiotis \\ National Technical University of Athens \\ School of Electrical and Computer Engineering \\ 9, Iroon Polytechniou St., 15773 Athens, Greece
}

\begin{abstract}
Many signal subspace-based approaches have already been proposed for determining the fixed Direction of Arrival (DOA) of plane waves impinging on an array of sensors. Two procedures for DOA estimation based neural network are presented. Firstly, Principal Component Analysis (PCA) is employed to extract the maximum eigenvalue and eigenvector from signal subspace to estimate DOA. Secondly, Minor component analysis (MCA) is a statistical method of extracting the eigenvector associated with the smallest eigenvalue of the covariance matrix. In this paper, we will modify a MCA learning algorithm to enhance the Convergence, where a Convergence is essential for MCA algorithm towards practical applications. The learning rate parameter is also presented, which ensures fast convergence of the algorithm, because it has direct effect on the convergence of the weight vector and the error level is affected by this value. MCA is performed to determine the estimated DOA. Simulation results will be furnished to illustrate the theoretical results achieved.
\end{abstract}

Index Terms-Direction of Arrival; Neural networks; Principle Component Analysis; Minor Component Analysis

\section{INTRODUCTION}

Neural networks have seen an explosion of interest over the last few years and are being successfully applied across an extraordinary range of problem domains, in areas as diverse as finance, medicine, engineering, geology, physics and biology. The excitement stems from the fact that these networks are attempts to model the capabilities of the human brain. From a statistical perspective neural networks are interesting because of their potential use in prediction and classification problems $[1,2,3]$. A neural network is an information-processing system that has certain performance characteristics in common with biological neural networks. Many methods for the estimation of the Direction of Arrival (DOA) have been proposed.

Dovid Levin et al in paper [4]: have explored the problem of SRP maximization with respect to a vector-sensor can be solved with a computationally inexpensive algorithm. A maximum likelihood (ML) DOA estimator is derived and sub subsequently shown to be a special case of DOA estimation by means of a search for the direction of maximum steered response power (SRP). The ML estimator achieves asymptotic efficiency and thus outperforms existing estimators with respect to the mean square angular error (MSAE) measure. The beampattern associated with the ML estimator is shown to be identical to that used by the minimum power distortion with less response beamformer for the purpose of signal enhancement.
Mitsuharu M. et al in paper [5]: have introduced the multiple signal classification (MUSIC) method that utilizes the transfer characteristics of microphones located at the same place, namely aggregated microphones. The conventional microphone array realizes a sound localization system according to the differences in the arrival time, phase shift and the level of the sound wave among each microphone. Therefore, it is difficult to miniaturize the microphone array.

Gao F. et al in paper [6]: have introduced a new spectral search-based direction-of-arrival (DOA) estimation method is proposed that extends the idea of the conventional ESPRIT DOA estimator to a much more general class of array geometries than assumed by the conventional ESPRIT technique.

In the context of DOA, the minor component is the direction in which the data have the smallest variance. Although eigenvalue decomposition or singular value decomposition can be used to extract minor component, these traditional matrix algebraic approaches are usually unsuitable for high-dimensional online input data. Neural networks can be used to solve the task of MCA learning algorithm [7]. Other classical methods involve costly matrix inversions, as well as poor estimation performance when the signal to noise ratio and number of samples are small and too large, respectively [8].

In many practical applications, a PCA algorithm deteriorates with decreasing signal to noise ratio and it may diverge in some cases to the learning rate giving incorrect results[9].For this reason, we need to handle this situation in order to overcome the divergence problem. In this context, we present a $\mathrm{MCA}(\mathrm{R})$ learning algorithm that has a low computational complexity. This allows the algorithm to update quickly (converge) to extract the smallest eigenvalue and eigenvector, which can be used to estimate DOA.

The paper is organized as follows. In Section II, we discuss the array signal model and we also describe a theoretical review of some existing Principal Component Analysis (PCA) and Minor Component Analysis (MCA) algorithms. In Section III, firstly we present the model for DOA measurements and then modified MCA algorithm is introduced. Finally in the same section, a convergence is analyzed. Simulations of results are included in Section IV to evaluate the convergence of the algorithm by comparison with aforementioned algorithms [10] and we verify our theoretical 
findings by comparing the algorithm results with the DOA. Finally, conclusions are drawn in Section V.

\section{SIGNAL MODEL AND LEARNING ALGORITHMS FOR PCA AND MCA}

\section{A. Signal Model}

Consider an array of omnidirectional sensors. The medium is assumed to be isotropic and non-dispersive. Since far-field source targets are assumed, the source wave front scan is approximated by plane waves. Then, for narrow band source signals, we can express the sensor outputs as the sum of the shifted versions of the source signals.

Consider a Uniform Linear Array (ULA) of $(m)$ omnidirectional sensors illuminated by $l$ narrow-band signals $(l<m)$. At the $l$ 'th snapshot the output of the $i$ 'th sensor may be described by [11]

$X=\sum_{i=1}^{d} \cos 2 \pi l d f_{i} \exp \left(\sqrt{-1} *(i-1) * 2 \pi \Delta \sin \left(\pi-\theta_{i}\right)\right.$

Where $\Delta$ is the space between two adjacent sensors, $\theta_{i}$ the angle of arrival, $d$ signals incident onto the array, $d f_{i}$ normalizes frequency. The incoming waves are assumed to be planned. The output of array sensors is affected by white noise which is assumed to be uncorrelated with the incoming signals. In vector notation, the output of the array results from $l$ complex signals can be written as:

$$
x(n)=c(\theta) s(n)+N(n)
$$

Where the vectors

$s(n)$ : signal vector , $N(n)$ : a noise vectorare defined as:

$x(n)=\left[x_{1}(\mathrm{n}), \ldots \ldots \ldots \ldots \ldots, \mathrm{x}_{m}(\mathrm{n})\right]_{m \times 1}^{T}$

$s(n)=\left[s_{1}(\mathrm{n}), \ldots \ldots \ldots \ldots \ldots, \mathrm{s}_{l}(\mathrm{n})\right]_{l \times 1}^{T}$

$N(n)=\left[N_{1}(\mathrm{n}), \ldots \ldots \ldots \ldots \ldots, \mathrm{N}_{m}(\mathrm{n})\right]_{m \times 1}^{T}$

And $C(\theta)$ is the matrix of steering vectors,

is the target DOA parameter vector,

$C\left(\theta_{i}\right)=\left[C\left(\theta_{1}\right), \ldots \ldots \ldots \ldots \ldots, C\left(\theta_{i}\right)\right]_{m \times l}$

Moreover,

$C(\theta)=\exp [-j 2 \pi i \sin \theta / v]$

$v=$ speedlight

\section{B. Learning Algorithm for PCA}

Consider the linear neural unit described by

$$
y(t)=w^{T} \cdot X(t) \text { where } X \in R
$$

Where the input vector, $w \in I R$ represents the weight vectors and $y$ denotes the neuron's output. The unit is used for extracting the first principal component from the input random signal, that is $y(t)$ should represent $X(t)$ in the best way, in the sense that the expectation error should be minimized.

$$
E_{\mathrm{x}}\left[\frac{\|x-y w\|^{2}}{w}\right]
$$

Here $E_{\mathrm{X}}[. / w]$ denotes mathematical expectation with respect to $x$ under the hypothesis $w$. The problem may be expressed as,

Solve: $\min E_{\mathrm{x}}\left[\|x\|^{2}-E_{\mathrm{x}}\left[\frac{y^{2}}{w}\right]\right.$ under $w w^{T}=1$
Consider the feed forward network shown in Fig.1. The following two assumptions of a structural network are made:

1) Each neuron in the output layer of the network is linear.

2) The network has $m$ inputs and l output, both of which are specified.Moreover the network has fewer outputs than inputs (i.e. $l<m$ ).

The only aspect of the network that is subject to training is the set of synaptic weights $w_{j i}$ connecting source nodes $i$, in the input layer to computation nodes $j$ in the output layer, where $i=0,1, \ldots \ldots, m$ and $j=0,1, \ldots . l$.

The output $y_{j}(n)$ of neuron $\mathrm{j}$ at time, produced in response to the set of inputs

$$
\begin{aligned}
& \left\{x_{i}(n)\right\}_{i=1}^{m}, \text { is given by } \\
& y_{j}(n)=\sum_{i=1}^{m} w_{j i}(n) x_{i}(n)
\end{aligned}
$$

The synaptic weight $w_{j i}$ is adapted in accordance with a generalized form of Hebbian learning [12,13] according to PCA as shown by:

$$
\Delta w_{j i}(n)=\eta\left[y_{j}(n) x_{i}(n)-y_{j}(n) \sum_{k=1}^{j} w_{k i}(n) y_{k}(n)\right]
$$

Where $\Delta w_{j i}(n)$, is the change applied to the synaptic weight $w_{j i}(n)$ at time, and $\eta$ is the learning rate parameter, greater than zero.

This principal component analysis algorithm has been found very useful for extracting the most representative lowdimensional subspace from a high-dimensional vector space. It is widely employed to analyze multidimensional input vector of hundreds of different stock prices, however when used in signal processing this algorithm deteriorates with decreasing signal to noise ratio [12].

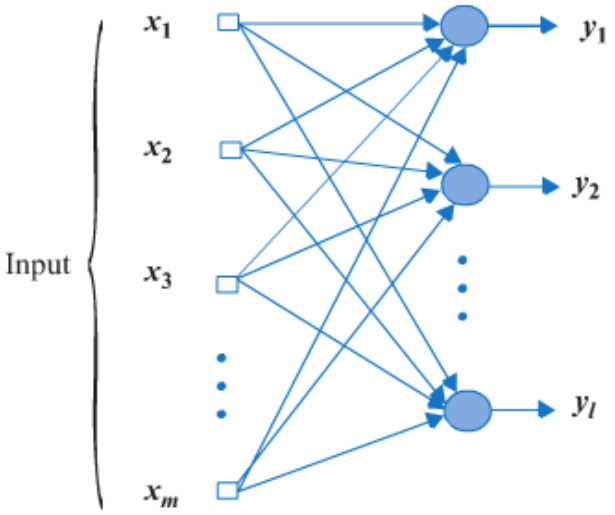

Fig. 1. Oja's single-layer linear neural network

\section{Learning Algorithm for MCA}

The opposite of PCA is Minor Component Analysis (MCA), is a statistical method of extracting the eigenvector associated with the smallest eigenvalue of the covariance matrix of input signals. As an important tool for signal processing and data analysis, MCA has been widely applied 
to: total least squares (TLS) [14], clutter cancellation [15], curve and surface fitting [16], digital beamforming [17], bearing estimation [18], etc. One single linear neuron can be used to extract minor component from input signals adaptively and the eigenvector associated with the smallest eigenvalue of the covariance matrix is called Minor Component, where one seeks to find these directions that minimize the projection variance. These directions are the eigendirections corresponding to the minimum eigenvalue. The applications of MCA arise in total least square and eigenvalue-based spectral estimation methods $[19,20]$. It allows the extraction of the first minor component from a stationary multivariate random process based on the definition of cost function to be minimized under right constraints. The extraction of the least principal component is usually referred to as MCA. For first Minor Component, what must be found is the weight vector that minimizes the power $E_{\mathrm{x}}\left[\frac{y^{2}}{\mathrm{~W}}\right]$ of neurons output.

For convenience, we produce a cost function for minor component estimation, that the problem is minimizing the cost function

$$
\begin{aligned}
\min _{w}\{J(\mathrm{w}) & =\frac{1}{2} E_{\mathrm{x}}\left[y^{2} / \mathrm{w}+\lambda / 2\left(\left(w^{\mathrm{T}} w-1\right)\right]\right\} \\
= & \frac{1}{2} E_{\mathrm{x}}\left[\left(\mathrm{w}^{\mathrm{T}} x\right)^{2} / \mathrm{w}+\lambda / 2\left(\left(w^{\mathrm{T}} w-1\right)\right]\right.
\end{aligned}
$$

With respect to the weight vector, its gradient has the expression,

$$
\frac{d j}{d w}=E_{x}[y x / w]+\lambda w
$$

Thus the optimal multiplier may be found by vanishing $w^{T} \frac{d j}{d w}$, that is by solving,

$$
\frac{d j}{d w}=E_{x}[y x / w]+\lambda w w^{T}=0
$$

Now the main point is to recognize that from an optimization point of view the above system is equivalent to:

$\frac{d j}{d w}=E_{x}[y x / w]+\lambda \beta\left(w w^{T}-1\right)=0, w w^{T}=1$

Where $\beta>0$, is a constant. It can be proven that the first minor converges to the expected solution providing that the $\operatorname{constant} \beta$ is properly chosen. This is the way to compute the optimal multiplier to obtain the stabilized learning rule [16]. The most exploited solution to the aforementioned problems consists of invoking the discrete-time versions of first minor, as

$$
\Delta w=-\eta\left[y x-y^{2} w\right]-\eta \beta\left(w w^{T}-1\right) w, w(0)=w_{0}
$$

Where $\eta$, is the learning rate and it's a common practice to make $\eta$ a sufficiently small value which ensures good convergence in a reasonably short time that represents the discrete time stochastic counterpart of first minor rules. Neural networks MCA learning algorithms can be used to adaptively update the weight vector and reach convergence to minor component of input data. In the first order the linear MCA will be:

$$
w_{i}(n+1)=w_{i}(n)-\eta y(n)\left[x_{i}(n)+y(n) w_{i}(n)\right]
$$

For a multiple output (neuron) the output $y_{j}(n)$ of neuron $j$,is produced in response to the set of input,

$$
x_{i}(n), i=0,1, \ldots, m
$$

And is given by,

$$
y_{j}(n)=\sum_{i=1}^{m} w_{j i}(n) x_{i}(n)
$$

The synaptic weight $w_{j i}$ is adapted in accordance with the generalized form of Hebbian, where the target of MCA is to extract the minor component from the input data by updating the weight vector $w(n)$ adaptively,

$$
\begin{aligned}
& \text { for all } w(n) \neq 0, \text { as, } \\
& \Delta w_{j i}(n)=-\eta\left[y_{j}(n) x_{i}(n)+y_{j}(n) \sum_{k=1}^{j} w_{k i}(n) y_{k}(n)\right]
\end{aligned}
$$

Where $\Delta w_{j i}(n)$, is the change applied to the synaptic weight $w_{j i}(n)$ at time, and Examining Eq.11, the term, $\eta y_{j}(n) x_{i}(n)$ on the right-hand side of the equation is related to Hebbian learning. As for the second term,

$$
\eta y_{j}(n) \sum_{k=1}^{j} w_{k i}(n) y_{k}(n)
$$

Is related to a competitive process that goes on among the synapses in the network. Simply put, as a result of this process, the most vigorously growing (i.e., fittest) synapses or neurons are selected at the expenses of the weaker ones. Indeed, it is this competitive process that alleviates the exponential growth in Hebbian learning working by itself. Note that stabilization of the algorithm through competition requires the use of a minus sign on the right-hand side of Eq.11. The distinctive feature of this algorithm is that it operates in a self-organized manner. This is an important characteristic of the algorithm that befits it for on-line learning. The generalized Hebbian Form of Eq.11, for a layer of neurons includes the algorithm of Eq.9, as

$$
w_{j i}(n+1)=w_{j i}(n)-\Delta w_{j i}
$$

Hence that,

$$
w_{j i}(n+1)=w_{j i}(n)-\eta\left[y_{j}(n) x_{i}(n)+y_{j}(n) w_{j i}(n)\right]
$$

\section{DOA MEASURMENT MODEL AND MCA MODIFIED ALGORITHM}

\section{A. DOA Model}

This algorithm uses measurements made on the signal received by an array of sensors. The wave fronts received by $m$ sensors array element are linear combination of incident waveforms $d$ and noises. The MCA begins with the following model of the received input data vector which is expressed as:

$$
\left[\begin{array}{c}
X_{1} \\
\cdot \\
X_{m}
\end{array}\right]=C\left(\theta_{1}\right), \ldots \ldots, C\left(\theta_{d}\right)\left[\begin{array}{c}
S_{1} \\
\cdot \\
S_{d}
\end{array}\right]+\left[\begin{array}{c}
N_{1} \\
\cdot \\
N_{m}
\end{array}\right]
$$


Where $S$, is the vector of incident signals, $N$ is the noise vector and $C\left(\theta_{d}\right)$ is the array steering vector corresponding to the DOA of the $i$ 'th signal. The received vector $X$ and the steering vector $C\left(\theta_{d}\right)$ as vector in $m$ dimensional space, the input matrix $\mathrm{R}_{\mathrm{xx}}$ can be expressed [21]:

$$
R_{x x}=E\left[X X^{T}\right]=E\left[S S^{T}\right] C C^{T}+E\left[N N^{T}\right]
$$

In many practical applications, the smallest eigenvalue of the matrix $R$ of input data is usually larger than zero due to the noisy signals. The column vectors of steering vectors, is perpendicular to the eigenvector corresponding to the noise. The $M C A$ spectrum may be expressed as,

$$
P_{M C A}\left(\theta_{d}\right)=1 /\left[C\left(\theta_{d}\right) w_{N} w_{N}^{T} C^{T}\left(\theta_{d}\right)\right]
$$

The matrix $w_{N} w_{N}^{T}$ is a projection matrix onto the noise subspace. For steering vectors that are orthogonal to the noise subspace, the denominator of Eq.16, will become very small and thus the peaks will occur in $P_{M C A}(\theta)$ corresponding to the angle of arrival of the signal. Where the ensemble average of the array input matrix $R$ is known and the noise can be considered uncorrelated and identically distributed between the elements [22].

TABLE I. A SUMMARY OF DIFFERENT DOA ALGORITHMS

\begin{tabular}{llll}
\hline \hline Method & $\begin{array}{c}\text { Power spectral as } \\
\text { function of, } \theta\end{array}$ \\
\hline \hline 1 & PCA & $C\left(\theta_{d}\right) R_{S S}^{-1} C\left(\theta_{d}\right)$ & Signal subspace \\
2 & MCA & $C\left(\theta_{d}\right) R_{N N} C\left(\theta_{d}\right)$ & Noise subspace
\end{tabular}

\section{B. The Modified MCA Algorithm}

The algorithm is based on MCA learning, which allows to update quickly and to extract the smallest eigenvalue and eigenvector, that can use these values to estimate DOA. The learning rate parameter is also presented, which ensures fast convergence of the algorithm.

To develop insight, the behavior of the GHA can be shown as: $\quad \hat{x}_{i}(n)=x_{i}(n)-\sum_{k=1}^{j} w_{k i}(n) y_{k}(n)$

In the last section, the weight vector yielded by GHA can further be modified by adding $\frac{1}{5 \eta} w$ to Hebbian rule (where the learning rate is often employed as small value) and a positive value $\varphi$, that is greater than the largest eigenvalue of matrix $R$. Recall from Section II-C, we can obtain the modified MCA algorithm as follows

$$
\begin{gathered}
w_{j i}(n+1)=1.2 w_{j i}(n) \\
\quad-\eta y_{j}(n)\left[\hat{x}_{i}(n)+\left(\varphi-y_{j}(n)\right) w_{j i}(n)\right] \\
=1.2 w_{j i}(n)-\eta y_{j}(n)\left[\hat{x}_{i}(n)+\left(\varphi-w_{j i}{ }^{T}(n) w_{j i}(n)\right) \hat{x}_{i}(n)\right]
\end{gathered}
$$

By taking $\varphi$ as $w_{j i}{ }^{T}(n) w_{j i}(n)-\frac{1}{5 \eta}$ for the modified MCA algorithm, and $\varphi>\lambda_{1}, 0<\eta \lambda_{\mathrm{i}}<1 / 5$, that is,

$$
=\left(1.2-\eta \lambda_{i}-\eta w_{j i}^{T}(n) w_{j i}(n)\right) w_{j i}(n)
$$

Convergence analysis needs to confirm the Eq.17, will converge to the minor component subject to the learning rate.

\section{Convergence Analysis}

In order to confirm the weight vector we will converge to minor component of input data in Eq.17 and it is important to discuss the learning rate $\eta$ because it has a direct effect on the convergence.

For convenience of analysis, since the matrix $R$ is a symmetrical nonnegative definite matrix.

The weight vector has unit length, that is

$$
\begin{gathered}
\|w\|^{2}=w w=1 \\
\left\|w_{j i}(n+1)\right\|^{2}=\left\|w_{j i}(n)\right\|^{2}+\left\|\Delta w_{j i}(n)\right\|^{2} \\
\geq\left\|w_{j i}(n)\right\|^{2} \\
w(n) w^{T}(n)=\|w(n)\|^{2} \geq\|w(0)\|^{2}>0,
\end{gathered}
$$

Let us assume that $\lambda_{1}, \ldots \ldots \ldots, \lambda_{i}$ be all the eigenvalues of matrix $R$, are ordered by,

$$
\lambda_{1}>\lambda_{2}>\cdots>\lambda_{i} \geq 0
$$

Where $w(0)$ is the initial weight vector, $\lambda_{1}$ is the largest eigenvalue of the matrix $R$ and the eigenvector associated with the smallest eigenvalue of $R$.

Suppose a definition of an invariant set $S$, as

The convergence analysis shows the learning rate suppose, $\eta \lambda_{1}<1 / 5$ and $\|w\|^{2} \leq 1 / 5 \eta$,

Where select $w(0)$ is the initial weight vector, $\lambda_{1}$ is the largest eigenvalue of the matrix $R$ and the eigenvector associated with the smallest eigenvalue of $R$, that

$1.2-\eta \lambda_{i}-\eta w w^{T} \geq 1.2-\eta \lambda_{1}-\eta w w^{T}$

$1.2-\eta \lambda_{i}-\eta w w^{T} \geq 1-\eta \lambda_{1}$

For $\eta \lambda_{1}<1 / 5$, it follows that

$1.2-\eta \lambda_{i}-\eta w w^{T} \quad \geq 0$

From $E q .17$, the condition is satisfied,

$1.2-\eta \lambda_{i}-\eta w w^{T} \geq 0$

$\left\|w_{j i}(n+1)\right\|^{2}=\sum\left[1.2-\eta \lambda_{i}-\eta w w^{T}\right]^{2}\|w(n)\|^{2}$

For $\|w\|^{2} \leq 1 / 5 \eta$,

$\leq\left[1.2-\eta\|w(n)\|^{2}\right]^{2}\|w(n)\|^{2}$

$\leq \max [1.2-\eta .1 / 5 \eta]^{2} 1 / 5 \eta$

$=1 / 5 \eta$

\section{Simulation RESUlts}

In this section we describe our simulation results. We will compare the convergence of our modified algorithm with aforementioned approaches by choosing a suitable learning rate where $\eta$ should satisfy $0<\eta \lambda_{1}<1 / 5$, Programs were written for DOA estimation in Matlab. A general test example is used for this purpose, with two sources, signal located at the far field at $\left(80^{\circ}, 120^{\circ}\right)$ degree with normalized frequencies of $(0.35,0.36)$ fs respectively were used. A ULA of five snapshots (L), eight sensors and sensor spacing equaling half wave length $(\Delta=0.5 \lambda)$, spacing was used to collect the data. 


\section{A. Simulation1: Effect of varying the learning rate parameter}

In this simulation we show that the effect of varying the learning rate parameter has a direct effect on the convergence of the weight vector. When the learning rate has a large step size that is shown in Fig.2, it allows the algorithm to update quickly, and may also cause the estimate of the optimum solution to wander significantly until the algorithm reaches convergence and the error reaches zero. When learning rate has a small step size that is shown, the convergence will be painfully slow typically. A small step size may be chosen to reduce this wandering until the desired accuracy is achieved but will require a long time for the algorithm to reach the optimum solution (fittest eigenvalue). Therefore, it should be selected a suitable learning rate in order to prevent learning divergence, because this unsuitable value will make the algorithm deviate drastically from the normal learning, which may result in divergence or an increased learning time.

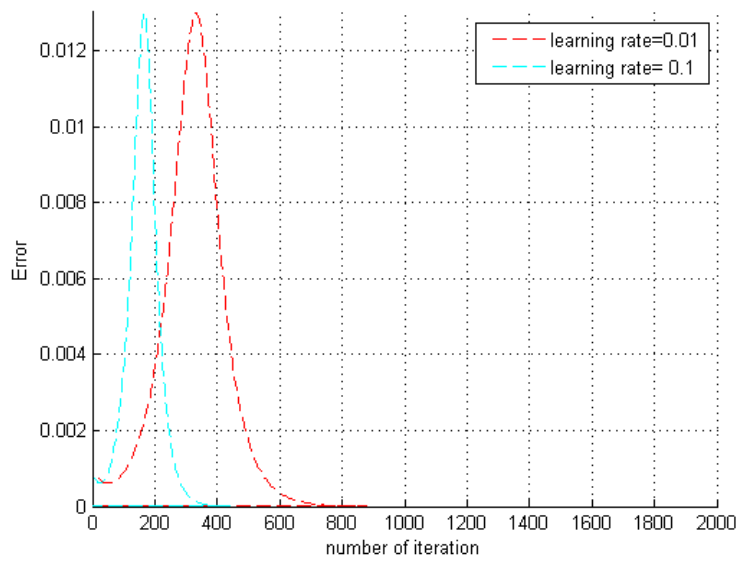

Fig. 2. Learning rate step when $\eta=(0.01$ and 0.1$)$

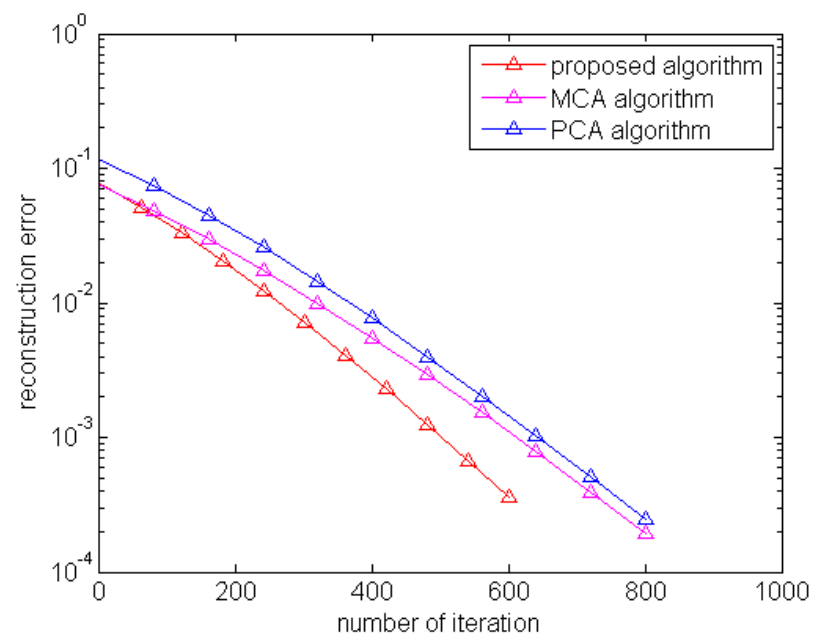

Fig. 3. Comparison convergence of algorithms

\section{B. Simulation 2: Comparison of methods with regard to convergence}

Fig.3, shows the Comparison convergence of the modified
MCA with aforementioned algorithms, that shows high performance of modified algorithm, where it has better convergence result than PCA and ordinary MCA algorithms. This is a result of choosing a more suitable learning rate, where the learning rate influences the overall rate of convergence. A smaller learning rate is selected.

\section{Simulation 3: Studying the performance effectiveness of the modified algorithm}

In this simulation in order to illustrate the effectiveness of the algorithm, we used measured DOA estimation based on the modified MCA to show the effectiveness of performance of this algorithm. It is a fact that we can obtain direction estimates better than PCA algorithm.

\section{1) Effect of Changing the Number of Snapshots}

- Figures (4,5) show the estimated DOA of incoming signal. It's apparent that the spectral peaks of modified MCA multiple sources become better when the number of snapshots is increased, as shown in Fig.5, when the number of snapshots is equal to five.

- Figures $(6,7)$ show the estimated DOA of two sources for incoming signals, with changing number of snapshot. Also, it is obvious that the spectral peaks of $P C A$ become sharp and the resolution increases when the number of snapshots is increased, as shown in Fig.7, when the number of snapshots is equals five.

\section{2) Effect of added white noise vector}

Figures $(8,9)$ show the estimated $D O A$ of two sources for incoming signals in $P C A$ and modified $M C A$, respectively, in order to compare a modified $M C A$ performance with $P C A$ when the input vector is affected by white noise vector. Fig.9, shows the modified $M C A$ estimate with right angles, where the spectral has better accuracy than the $P C A$ spectral plotted as shown in Fig.8.

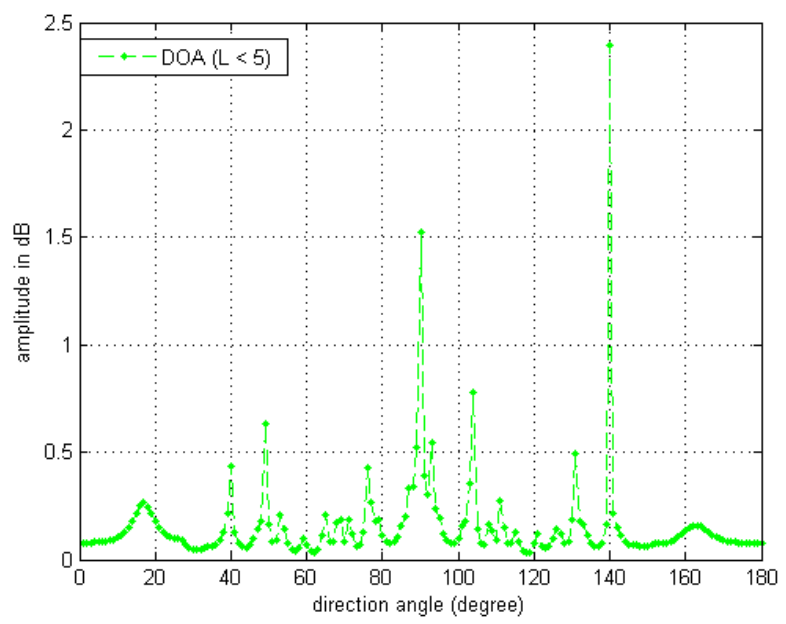

Fig. 4. Estimation $D O A$ by modified $M C A$ when number of snapshots $L<5$ 


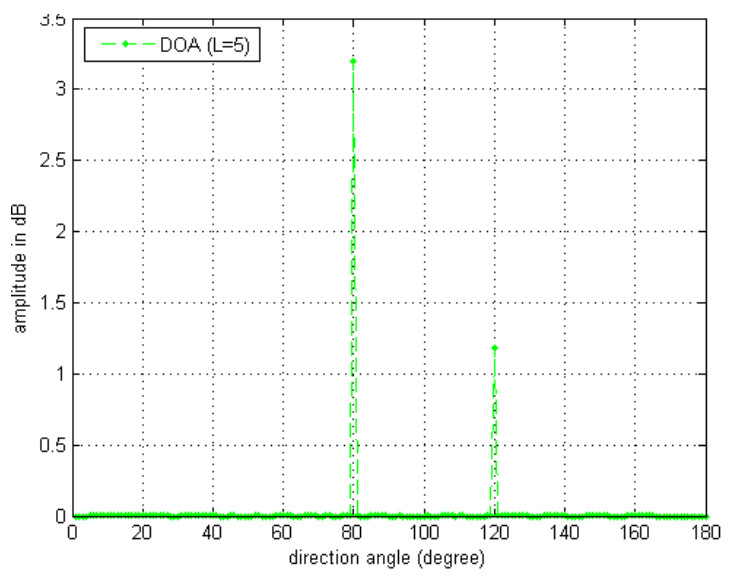

Fig. 5. Estimation $D O A$ by modified $M C A$ when number of snapshots $L=5$

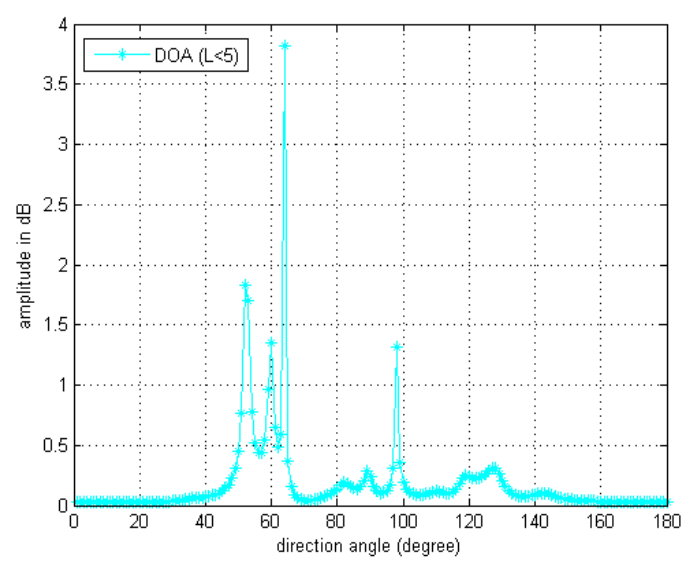

Fig. 6. Estimation $D O A$ by $P C A$ when number of snapshots $L<5$



Fig. 7. Estimation DOA by $P C A$ when number of snapshots $L=5$

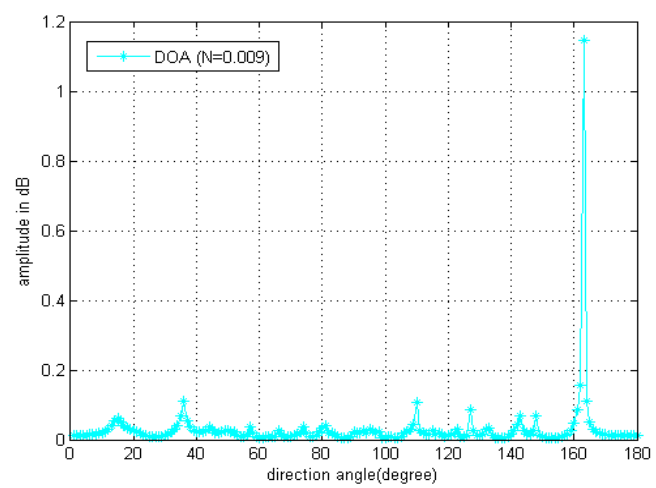

Fig. 8. Estimation $D O A$ by $P C A$ when additive noise $N=0.009 d B$

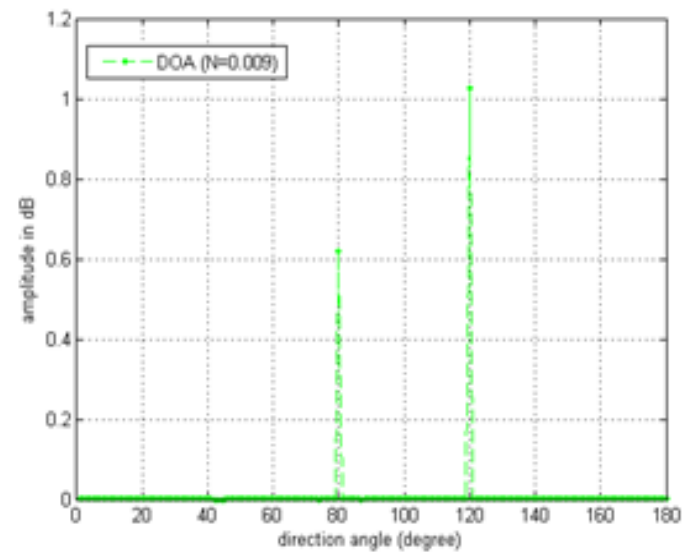

Fig. 9. Estimation DOA by modified $M C A$ when additive noise $N=0.009$ $d B$

\section{CONCLUSION}

This paper presented a prototype direction of arrival estimation. During this study, a simple MCA learning algorithm is presented to extract minor component from input signals to enhance the convergence. The learning rate parameter is also presented which ensures fast convergence of the algorithm. Clearly, this shows that the modified MCA has quickly converged to the minor component subjected to the learning rate. In this context, the learning rate usually should be set at a suitable value to reach the optimum solution and to move the algorithm too close in the "correct" direction.

Also, this demonstration shows that the modified MCA algorithm achieves to produce a right angle $\theta$ for the DOA, when the input vector is affected by white noise vector better than the PCA algorithm that fails to produce a value for the DOA above a certain level of noise. 
The main advantage of this algorithm is it can better tolerate noises signals to extract the minimum eigenvalue from noise subspace and it has been applied to find DOA estimation.

\section{ACKNOWLEDGMENT}

This research is supported by the School of Electrical and Computer Engineering, National Technical University in Athens (NTUA), Greece

\section{REFERENCES}

[1] Alexander I., Galushkin "Neural Networks Theory"Springer-Verlag Berlin Heidlberg, 2007, ISBN 0-387-94162-5.

[2] Timo Honkela, Włodzisław Duch." Artificial Neural Networks and Machine Learning -ICANN 2011" 21st International Conference on Artificial Neural Networks Espoo, Finland, June 14-17, 2011 Proceedings.

[3] G. Dreyfus."Neural Networks, Methodology and Applications" Original French edition published by Eyrolles, springer, Paris,2004, ISBN 103540-22980.

[4] Dovid Levin, Emanuel A., Sharon G. "Maximum Likelihood Estimation of Direction of Arrival using an acoustic vector-sensor", International Audio Laboratories Erlangen, Germany, 2012.

[5] Mitsuharu M., Shuji H. "Multiple Signal Classification by Aggregated Microphones" 2005, IEICE, ISSN: 0916-8508.

[6] FeifeiGao and Alex B. Gershman "A Generalized ESPRIT Approach to Direction-of-Arrival Estimation”IEEE Signal Processing Letter, Vol. 12, No. 3, March 2005.

[7] Rashid Ahmed, John A. Avaritsiotis," MCA Learning Algorithm for Incident Signals Estimation:A Review”, IJCTT Journal,Feb. 2014.

[8] G.Wang and X.-G.Xia." Iterative Algorithm for Direction of Arrival Estimation with wideband chirp signals" IEEE., 2000, ISSN : 13502395.

[9] Weidong J., Shixi Y., Yongping C.," DOA Estimation of Multiple Convolutively Mixed Sources Based on Principle Component Analysis", Springer, ICONIP , pp. 340-348, 2009.

[10] Qingfu Zhang, Yiu-Wung Leung, "A Class of Learning Algorithms for Principal Component Analysis and Minor Component Analysis", IEEE Transection on Neural Network, Vol. 11, No.2, March 2000.

[11] Adnan S.," DOA Based Minor Component Estimation using Neural Networks",AJES, Electrical Engineering Dept., Vol.3, No.1, 2010.

[12] Yanwa Zhang "CGHA For Principal Component Extraction In The Complex Domain “,IEEE, Transaction on Neural Networks ,Vol. 8,no. 5,pp. 1031-1036, sept. 1997.
[13] Kwang In Kim, Matthias O. Franz, Bernhard, "Kernel Hebbian Algorithm for Iterative Kernel Principal Component Analysis ”,Max Planck Institute for Biological Cybernetics, June 2003.

[14] K. Gao, M.O. Ahmad, M.N. Swamy, "Learning Algorithm for Total Least Squares Adaptive Signal Processing, Electronics Letters. Feb. 1992.

[15] S. Barbarossa, E. Daddio, G. Galati, "Comparison of Optimum and Linear Prediction Technique for Clutter Cancellation", Communications, Radar and Signal Processing, IEE Proceedings, ISSN (0143-7070).

[16] L. Xu, E. Oja, C. Suen, "Modified Hebbian Learning for Curve and Surface Fitting, Neural Networks, 1992.

[17] J.W. Griffiths, "Adaptive Array Processing, A Tutorial, Communications, Radar and Signal Processing, IEE Proceedings, ISSN :0143-7070.

[18] R. Schmidt, Multiple Emitter Location and Signal Parameter Estimation, IEEE Trans. Antennas Propagation (1986) 276-280.

[19] Dezhong Peng, Zhang Yi." A New Algorithm for Sequential Minor Component Analysis" International Journal of Computational Intelligence Research ,ISSN 0973-1873 Vol.2, No.2 (2006).

[20] Jie Luo, Xieting Ling "Minor Component Analysis with Independent to Blind 2 Channel Equalization," IEEE, Fudan University-China.

[21] Donghai Li, Shihai Gao, Feng Wang, "Direction of Arrival Estimation Based on Minor Component Analysis Approach", Neural Information Processing, Springer-Verlag Berlin Heidelberg, 2006.

[22] Belloni F., Richter A., Koivunen V. " DOA Estimation via Manifold Separation for Arbitrary Array Structures," IEEE, Transaction on signal processing, Vol, 55,No.10, October, 2007.

\section{AUTHOR PROFILE}

Rashid Ahmed is a Ph.D student in the School of Electrical and Computer Engineering, National Technical University of Athens-NTUA, Greece, and is a student member of the IEEE.

John N. Avaritsiotis is a Professor of Microelectronics in the Department of Electrical and Computer Engineering of the National Technical University of Athens-NTUA. He has published over 80 technical articles in various scientific journals, and has presented more than 30 papers at international conferences. His present research interests concern the development of surface micro-machining processes for the production of micromechanical sensors and design and prototyping of various types of multi-sensor systems for various applications. He is the Director of two R\&D Laboratories: the Microelectronics Lab and the Electronic Sensors Lab of NTUA. He is the Co-Editor of the Journal Active and Passive Electronic Devices, Guest Editor of IEEE Transactions on Components, Packaging and manufacturing Technology, Senior Member of IEEE and Member of IOP and ISHM. 